\title{
Hallazgo de un triente emeritense de la serie LATINA MVNITA en Cáceres (España)
}

\section{Finding of an emeritensis tremissis of the}

LATINA MVNITA series in Cáceres (Spain)

\author{
Alberto González García \\ Universidad Complutense de Madrid \\ agonza08@ucm.es
}

David Martínez Chico

Universitat de València

damarchi@alumni.uv.es

\section{Resumen}

El artículo documenta el hallazóo de un triente suevo con leyenda LATINA EMERI MVNITA en el norte de la provincia de Cáceres (España). Su localización refuerza el tradicional origen atribuido a esta tipología, en la ceca de Emerita y no en territorio gallego. Se discute la naturaleza y cronología de estas piezas.

\section{Palabras clave}

Suevos, Moneda, Cecas, Emerita, Tardoantigüedad.

\begin{abstract}
The paper documents the finding of a suebi triens with legend LATINA EMERI MVNITA in the north of the province of Cáceres (Spain). Its location reinforces the traditional origin attributed to this typology, in the mint of Emerita and not in the Galician territory. The nature and chronology of these pieces are discussed.
\end{abstract}

\section{Keywords}

Suebi, Coinage, Mints, Emerita, Late antiquity.

Data de envío: 27-04-2017 Data de aceptación: 30-05-2017 


\section{INTRODUCCIÓN Y DESCRIPCIÓN}

Nuestro deseo con la siguiente nota es dar a conocer el hallazgo de un curioso tremís de la serie LATINA MVNITA, atribuida de forma dudosa a la monarquía sueva, y datada en el s. VI, procedente de la ceca de Emerita Augusta (Figura 1). La pieza, según testimonio de su propietario original, fue descubierta en un punto indeterminado de la provincia de Cáceres, probablemente en la mitad norte. Se subastó en un célebre portal de ventas online en marzo de 2017, alcanzando un considerable precio. Tratándose una tipología extremadamente escasa, ya que sólo se documentan otros cuarenta y un ejemplares (CABRAL-METCALF 1997: 89-97; ROMA 2002: 79; CEBREIRO 2012: 48-54), siendo éste uno de los pocos con leyenda LATINA EMERI MVNITA (existe otro con la variante LATINA EMERI MIONIT), y dada su importancia para la historia monetaria del reino suevo, creemos que merece la pena dedicar unas líneas a esta moneda. La descripción de la pieza es la que sigue.
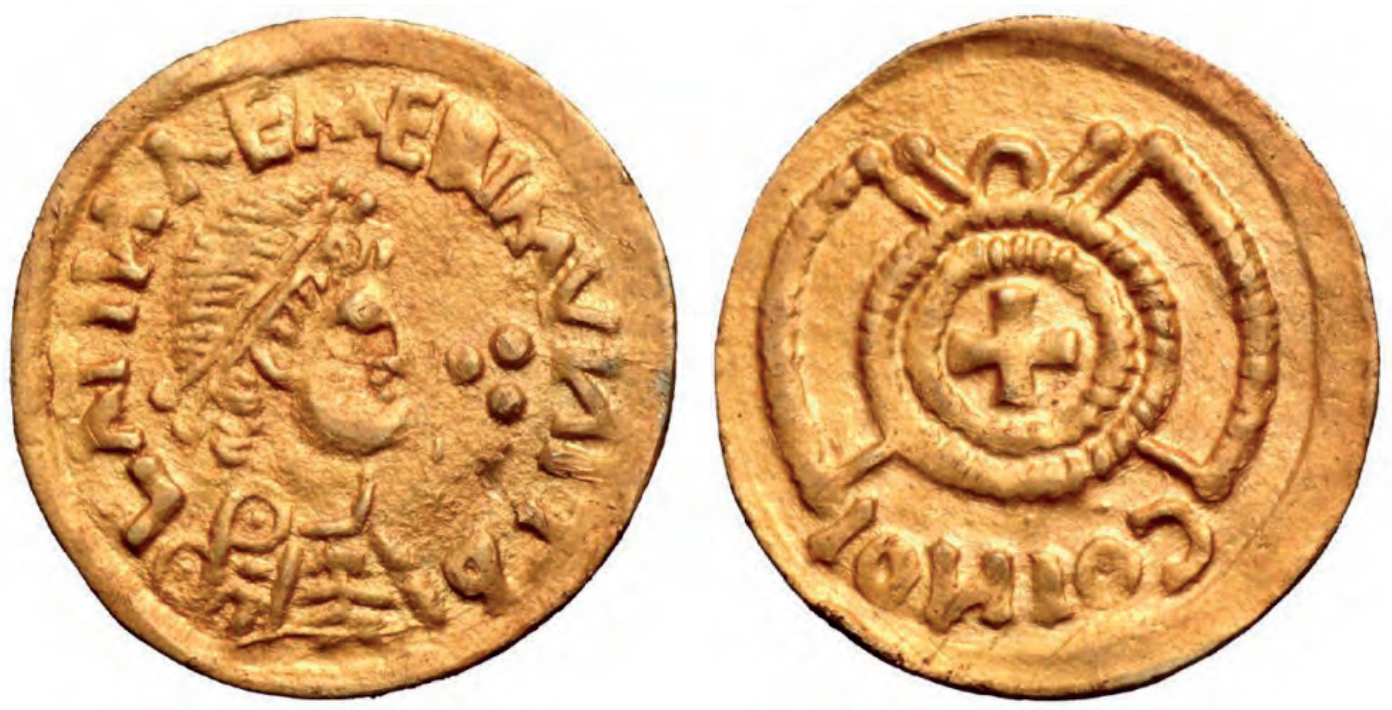

Figura 1. Tremis suevo acuñado en Mérida.

Anv.: LATINA EMERI MVNITA / busto diademado, acorazado y drapeado con fíbula enjoyada a derecha / campo derecho: tres puntos dispuestos en triángulo equilátero con vértice a izquierda, frente a la boca del busto.

Rev.: cruz equilátera inscrita en una corona de laurel doble, flanqueada por dos trapecios circulares / exergo: COINOI (retrógrado).

Datos: $1.17 \mathrm{~g}, 17 \mathrm{~mm}, 5 \mathrm{~h}$.

Referencia: REINHART 1937: 186, 50; PEIXOTO-METCALF 1997: 300, 1; cf. GOMES 2003: 45, 02.11.

1 Roma Numismatics Auction XIII, lote 1018: < http://romanumismatics.com/auction/lot/1018/> [Consultado: 11-3-2017]. 


\section{ACERCA DE LA SERIE LATINA MVNITA}

Los tremisses del tipo LATINA MVNITA se caracterizan por un peso medio levemente inferior al teórico, un contenido medio de oro en torno al 70-80\%, y una concentración de hallazgos en el norte del actual Portugal (CABRAL-METCALF 1997: 86, 121-122 y 152-154; ROMA 2002: 80-81). El cuidado técnico y las similitudes entre unos y otros ejemplares hace pensar en que fueron producidos por monederos itinerantes; estilísticamente, se distinguen tres grupos, uno bergidense (al que pertenecen las LATINA EMERI MVNITA), otro legionense y un tercero que calificaron como "indeterminado", con leyendas de complicada lectura (PEIXOTO-METCALF 1997: 89-97; METCALF 1998; ROMA 2012: 81-82), sin que haya una relación clara con sus supuestas cecas de origen.

Su peso es muy variable, en un rango de 0.79 a $1.42 \mathrm{~g}$, con una media de 1.11 , su contenido de oro se sitúa entre el 62 y el 86\%, y la media en 75.7\% (PEIXOTO-METCALF 1997: 131-132 y 176-178).

Sobre el origen concreto del metal que las compone, sin un análisis del mismo para determinar sus características y origen, cualquier comentario es puramente especulativo. Se suele datar el agotamiento y abandono de las minas de oro del noroeste peninsular en el final del período severiano (DOMERGUE 1990: 215-223 y 313-316), y, aunque hay juicios más optimistas sobre la minería tardoimperial y post-romana (REINHART 1952: 134; KING 1981: 219-220; EDMONSON 1989; QUIROGA-LOVELLE 1999), y la cerámica bizantina peninsular se concentre en los tradicionales centros de producción mineral, también en el noroeste (DARK 1996: 209-213), carecemos de datos que nos permitan valorar la actividad minera en época sueva, y mucho menos aún la extracción o bateo de oro. Por ello estimamos ocioso entrar en la cuestión.

La naturaleza de estas emisiones ha provocado una multiplicidad de opiniones. Por un lado, se encuentran los autores que defienden un origen provincial y municipal para el conjunto, diferenciado de la monarquía sueva (BELTRÁN 1960; GRIERSONBLACKBURN 2006: 79-80). Gomes Marques y Cardim Ribeiro (GOMES 1998; 168-169; GOMES-CARDIM 1998) concluyeron, mediante análisis filológico, que las leyendas representan en muchos casos nombres en genitivo de los monederos, considerando que no hay datos que permitan determinar su origen, y mucho menos adscribirlas a la monarquía sueva. Para otros (MARTÍN VISO 2008: 244-245; 2011: 195-200), esos nombres corresponderían a notables locales con amplios recursos y autonomía.

Por otro lado, tenemos a los partidarios de que estas piezas fueron acuñadas por los reyes suevos, situando la serie en el marco de los enfrentamientos militares entre suevos y visigodos durante los ss. Vy VI, donde las monedas de origen emeritense se explicarían más fácilmente, estimando Livermore su origen en la autonomía del poderoso obispo Masona, c. 573-606 (REINHART 1937; LIVERMORE 1990; CABRAL-METCALF 1997).

La polémica estéril referente a la naturaleza de la autoridad emisora, y la falaz y radical dicotomía entre el poder municipal y el real puede romperse recordando la existencia de la figura del comes civitatis, máxima autoridad urbana con autonomía y amplios poderes civiles, fiscales y militares, pero delegada del monarca, que fue característica de las ciudades tardoantiguas de Hispania y la Galia (GARCÍA 1974: 8-12). 
Nuestra moneda presenta tres puntos muy llamativos, que también se observan, junto con otras muchas marcas, en otras piezas suevas (CABRAL-METCALF 1997, passim). Podríamos considerarlos marcas de control, obra de los monederos, dada la proliferación de signos similares en la moneda altomedieval, y su temprana presencia en piezas visigodas (e.g. BARTLETT-CORES-CORES 2005; GRIERSON-MAYS 2006: 52).

La localización del hallazgo en territorio de Cáceres, provincia cercana a Mérida, hace admisible seguir aceptando el tradicional origen emeritense, no exento de polémica, para esta tipología (REINHART 1937 y 15952: 132-134; MATEU 1942; BELTRÁN 1958; CABRAL-METCALF 1997; METCALF 1998), ya que otros autores (GOMES 1998, 173-185; GOMES-CARDIM 1998; MARTÍN 2008: 244-245; MARTÍN 2011: 195-200) estiman la leyenda EMERI como correspondiente al nombre propio de un monedero o potentado local (e.g. Emerius), en vez de a la ciudad de Emerita.

Cabe destacar que la rica Mérida, antigua capital diocesana hispánica e importante obispado, fue objeto de considerables disputas durante el siglo V. Tomada por los suevos en 439 (junto con Mértola), se convirtió en una pieza clave de su reino hasta su caída en manos visigodas en 456, y quizállegaran a reconquistarla temporalmente en algún momento entre los años 470 y 510, como sugieren ciertos indicios (DÍAZ 2011: 76-83 y 119-121).

Además de la que aquí presentamos, las piezas de este tipo más próximas a la ciudad de Mérida son el triente con leyenda MVNITA GALLICA PAX localizado en Castelo Branco, y el LATINA EMERI MVNITA de Lisboa (REINHART 1937: 186, 49 y 51; BARRAL 1976: 167). También contamos con otras piezas suevas próximas a la provincia de Cáceres. En Badajoz, Castelo Branco, Covilha, Coimbra y Portalegre tenemos cinco sólidos imitativos a nombre de Honorio (REINHART 1937: 182, 1y 3; REINHART 1942: 321, 6 y 7; MARQUES 1988: 71-72; PEIXOTO-CABRAL 1997: 207); en Castelo Branco, en Évora, en Castelho Velho (Rochoso, Guarda), y en Vermelha (Alcobaça) cuatro trientes a nombre de Valentiniano III (REINHART 1937: 184, 24; VASCO 1959: 27; MARQUES 1988: 72; BARRAL 1976: 166; PEIXOTO-CABRAL 1997: 207-8); otros dos tremisses de leyendas ininteligibles en Castelo Branco (REINHART 1937: 188, 70 y 71; BARRAL 1976: 167); y, finalmente, una silicua de Requiario en Alcáçova de Santarém (CEBREIRO 2012: 58). Indicamos la localización de todas estas poblaciones en el subsiguiente mapa (Figura 2).

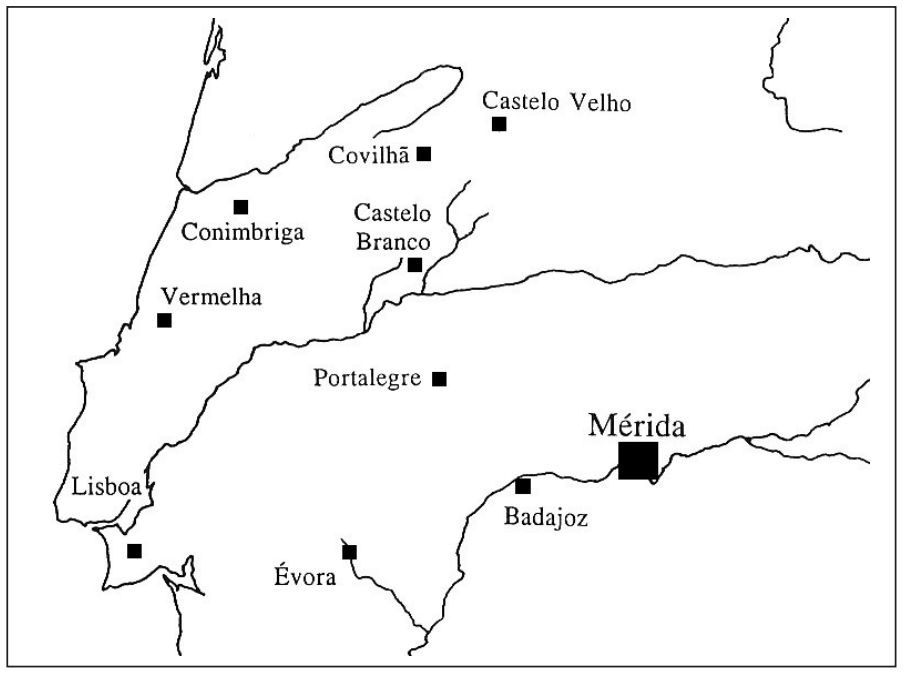

Figura 2. Mapa de situación de Mérida y localizaciones de las monedas suevas en sur de Portugal y Extremadura. 
En los últimos años, en el norte de Cáceres se han producido diversos hallazgos fortuitos de nuevos materiales numismáticos tardorromanos y visigodos que evidencian la riqueza de la antigua Lusitania y un importante nivel de monetización en la Antiguedad Tardía, vinculado a la capital diocesana y a la Vía de la Plata (e. g. MARTÍNEZ 2014; MARTÍNEZ-LÓPEZ 2017; MARTÍNEZ-GONZÁLEZ 2017).

Finalmente, con respecto a la cronología, hay que considerar sus similitudes tipológicas con las imitaciones de Valentiniano III (ya señaladas por REINHART 1937; BARRAL, 1976: 49; CARSON-KENT-BURNETT 1994, 230-231; GRIERSON-BLACKBURN 2006: 77-80), el ante quem marcado por el tesorillo visigodo hallado en Recópolis, datado en 576-579 (BARRAL 1976: 86-92) y el hecho de que no se repite ningún cuño en los ejemplares conocidos, a excepción de un ejemplar EMERI cuyo reverso se fabricó con el mismo cuño que un triente de imitación de Valentiniano III, y que tiene idéntico contenido metálico, lo cual significa que debieron ser inmediatamente posteriores, como bien defiende Roma Valdés (PEIXOTO-METCALF 1997: 85 y 103-104; ROMA 2002: 81 y 84).

Heiss (1891) diferenciaba entre emisiones gallegas y lusitanas, proponiendo tres períodos de acuñación: un primero gallego (411-56), correspondiente a imitaciones a nombre de Honorio y Valentiniano III, un segundo lusitano (430-57), con las piezas LATINA EMERI MVNITA y LATINA MVNITA y un tercero nuevamente gallego (457-84), con imitaciones de tremisses más degradadas.

Actualmente se estima que los suevos comenzaron a acuñar moneda a partir de 45253, momento en el que se realiza un nuevo pacto con el Imperio, devolviendo la Cartaginense y conservando Lusitania (DÍAZ 2011: 80-81; GOMES 1998: 112). A esta época corresponderían las silicuas de Requiario (448-56) en nombre del difunto Honorio y las imitaciones de sólidos de este mismo emperador, pero no hay evidencias que permitan atribuir las imitaciones de Valentiniano III al siglo VI, y López Sánchez piensa que pueden corresponder más bien a la década de 440 (LÓPEZ 2010).

En consecuencia, consideramos rechazable la teoría que atribuye a las LATINA MVNITA una cronología tardía, exclusiva del último tercio del siglo VI, coincidiendo con la conversión al catolicismo de los suevos y una presunta expansión, y a las imitaciones de Valentiniano III una larguísima cronología, de 455 a 559 (LIVERMORE 1990; METCALF 1993; PEIXOTO-METCALF 1997: 25-26, 69, 94-96 y 184; METCALF 1998; GARCÍA 2006; DÍAZ 2011: 203-204).

Como se ha visto, existe un ejemplar EMERI cuyo reverso se fabricó con el mismo cuño que un triente de imitación de Valentiniano III. Si estas imitaciones tienen una cronología temprana, a mediados del V, las posibles emisiones póstumas, no pueden ser en exceso posteriores. Por ello estimamos más plausible adjudicar el inicio de la emisión de las LATINA MVNITA a la segunda mitad del s. V, y limitarlas no a finales del VI, lo cual genera toda clase de problemas y paradojas, empezando por la necesaria exclusión de un origen emeritense para piezas como la que nos ocupa, y la búsqueda de alternativas poco convincentes. 


\section{Conclusiones}

El hallazgo en territorio cacereño de un nuevo ejemplar de tremissis del tipo LATINA EMERI MVNITA aumenta un corpus escasísimo.

Su localización apunta a que su supuesto origen emeritense nada tiene de descabellado, restando crédito a la identificación de la palabra EMERI con el nombre de un monedero o prócer local.

Respecto a la cronología, debido a la propia la iconografía y la existencia de un cuño compartido con imitaciones de Valentiniano III, estimamos más lógico adjudicar estas piezas a la segunda mitad del siglo V, y no a finales del VI.

\section{Bibliografía}

BARRAL I ALTET, X. 1976. La circulation des monnaies Sueèves et Visigothiques. Contribution à l'historire économique du royaume visigot. Beihefte der Francia, vol. 4. Múnich.

BARTLETT, P., CORES URÍA, G., CORES GOMENDIO, M.C. 2005. "The Use of Dots as Control Marks in the Coin Legends at the Visigothic Mint of Ispali during the Reign of Sisebut (612-621)". En Actas del XIII Congreso Internacional de Numismática (septiembre 2003). Madrid, t. II, pp. 11271133.

BELTRÁN VILLAGRASA, P. 1960. "Interpretaciones de algunas monedas suevas", NVMMVS, ser. 6, 20-22, pp. 81-90.

CARSON, R.A.G., KENT, J.P.C., BURNETT, A. 1994. The Roman Imperial Coinage, vol. X: The Divided Empire and the Fall of the Western Parts, AD 395-491. Londres.

CEBREIRO ARES, F. 2012. "Dificultades que plantea el estudio de la historia monetaria sueva". En Cebreiro Ares, F. (ed.), Introducción a la historia monetaria de Galicia (s. II a.C. - XVII d.C.). La Coruña, pp. 31-63.

DARK, K.R. 1996. External Contacts and the Economy of Late Roman and Post-Roman Britain. Woodbridge.

DÍAZ MARTÍNEZ, P.C. 2011. El reino suevo (411-585). Madrid.

DOMERGUE, C. 1990. Les mines de la péninsule Ibérique dans l’Antiquité romaine. Roma.

EDMONSON, J.C. 1989. "Mining in the Later Roman Empire and Beyond: Continuity or Disruption?", J ournal of Roman Studies, 79, pp. 84-102.

GARCÍA MORENO, L.A. 1974. "Estudios sobre la organización administrativa del reino Visigodo de Toledo", Anuario de Historia del Derecho Español, 44, pp. 5-155.

GARCÍA MORENO, L.A. 2006. "La Iglesia y el Cristianismo en la Galecia de época sueva", Antigüedad y cristianismo, 23 (Ejemplar dedicado a: Espacio y tiempo en la percepción de la antigüedad tardía: homenaje al profesor Antonino González Blanco, "In maturitate aetatis ad prudentiam”), pp. 39-56.

GOMES MARQUES, M. 1998. A moeda peninsular na Idade das Trevas. Sintra.

GOMES MARQUES, M., CARDIM RIBEIRO, J. 1998. “As legendas da série Latina Munita”, en IV Congresso Nacional de Numismática, Lisboa, 23 a 25 de J ulho de 1998. Lisboa, pp. 69-98.

GRIERSON, P., BLACKBURN, M. 2006. Medieval European Coinage 1, The Early Middle Ages (5th10th Centuries). Cambridge.

HEISS, A. 1891. «Essai sur le monnayage des Suèves», Revue numismatique, ser. 3, t. 9, pp. 146-163. KING, P.D. 1981: Derecho y sociedad en el reino visigodo de Toledo. Madrid.

LIVERMORE, H.V. 1990. "The Coinage of the Suevic Period", NVMMVS, ser. 2., 12-13, pp. 39-49. 
LÓPEZ SANCHEZ, F. 2010. "Suevic Kings and Suevic Coins: The Visigothic Connection”. En Curta, F. (ed.), Neglected Barbarians. Turnhout, pp. 503-536.

MARQUES DE FARIA, A. 1988. "On finds of Suevic and Visigothic coins in the Iberian Peninsula and Their Interpretation”, Problems of Medieval Coinage in the Iberian Area 3. Santarem, pp. 71-88.

MARTÍN VISO, I. 2008. "La ordenación del territorio rural y la tributación en el suroeste de la meseta del Duero (siglos VI-VII)”. En Castellanos, S. y Martín Viso, I. (ed.), De Roma a los bárbaros. Poder central y horizontes locales en la cuenca del Duero. León, pp. 227-261.

MARTÍN VISO, I. 2011. "Circuits of Power in a Fragmented Space: gold coinage in the Meseta del Duero (Sixth-Seventh Centuries)”. En Escalona, J. y Reynolds, A. (eds.), Scale and scale change in the Early Middle Ages. Exploring landscape, local society and the world beyond. Turnhout, pp. 215-252.

MARTÍNEZ CHICO, D. 2014. "Notas sobre un peculiar numisma imitativo de Constancio II hallado cerca de la Vía de la Plata”, Acta numismàtica, 44, pp. 159-165.

MARTÍNEZ CHICO, D., LÓPEZ SÁNCHEZ, F. 2016. «Une nouvelle variété de solidus au nom de Constance II avec le chrisme à l'intérieur du bouclier», Bulletin de la Société Française de Numismatique, 71/4 (abril), pp. 138-141.

MARTÍNEZ CHICO, D., GONZÁLEZ GARCÍA, A. 2017 (en prensa). «Nuevos hallazgos monetales visigodos. Oro y bronce en el norte de Cáceres», Habis, 48.

MATEU Y LLOPIS, F. 1942. "Los nombres de lugar en el numerario suevo y visigodo de la Gallaecia y Lusitania (Notas para su estudio)”, Analecta Sacra Tarraconensia, 15, pp. 23-42.

METCALF, D.M. 1993. "The Coinage of the First and Second Suevic Kingdoms: from romanitas to latinization”. En Galicia: da romanidade á xermanización. Problemas históricos e culturais (Actas do encontro científico en homenaxe a Fermín Bouza Brey (1901-1973): Santiago de Compostela, outubro 1992). Santiago de Compostela, pp. 355-365.

METCALF, D.M. 1998. “Where Were Suevic Coins Minted?”. En IV Congresso Nacional de Numismática, Lisboa, 23 a 25 de Julho de 1998. Lisboa, pp. 57-68.

PEIXOTO CABRAL, J.M. y METCALF, D.M. 1997. A moeda sueva. Suevic Coinage (Anexos NVMMVS, no. 4). Oporto.

QUIROGA, J.L. y LOVELLE, M.R. 1999. "Cecas y hallazgos monetarios de época suevo-visigoda: civitates y vías de comunicación en el noroeste de la península ibérica”. En Centeno, R.M.S, García Bellido, M.P. y Mora, G. (coords.), Rutas, ciudades y moneda en Hispania: actas del II Encuentro Peninsular de Numismática Antigua Porto, marzo de 1997. Madrid, pp. 433-440.

REINHART, W. 1937. “Die Münzen des Schwebenreiches”, Mittailungen der Bayerischen Numismatiischen Gesellschaft, 55, pp. 151-190.

REINHART, W. 1942. "El reino hispánico de los suevos y sus monedas", Archivo español de arqueología, 15, pp. 308-328.

REINHART, W. 1952. Historia general del reino hispánico de los suevos. Madrid.

ROMA VALDÉS, A. 2002. "Emisiones monetarias del siglo VI d.C. con leyenda LATINA MVNITA. Estado del debate”, Brigecio, 12, pp. 79-84.

VASCO RODRIGUES, A. 1959. "Subsídios numismáticos para o estudo da dominação suévico-visigótica na região da Guarda (Elementos inéditos)”, Bracara Augusta, 9-10, pp. 25-29. 